
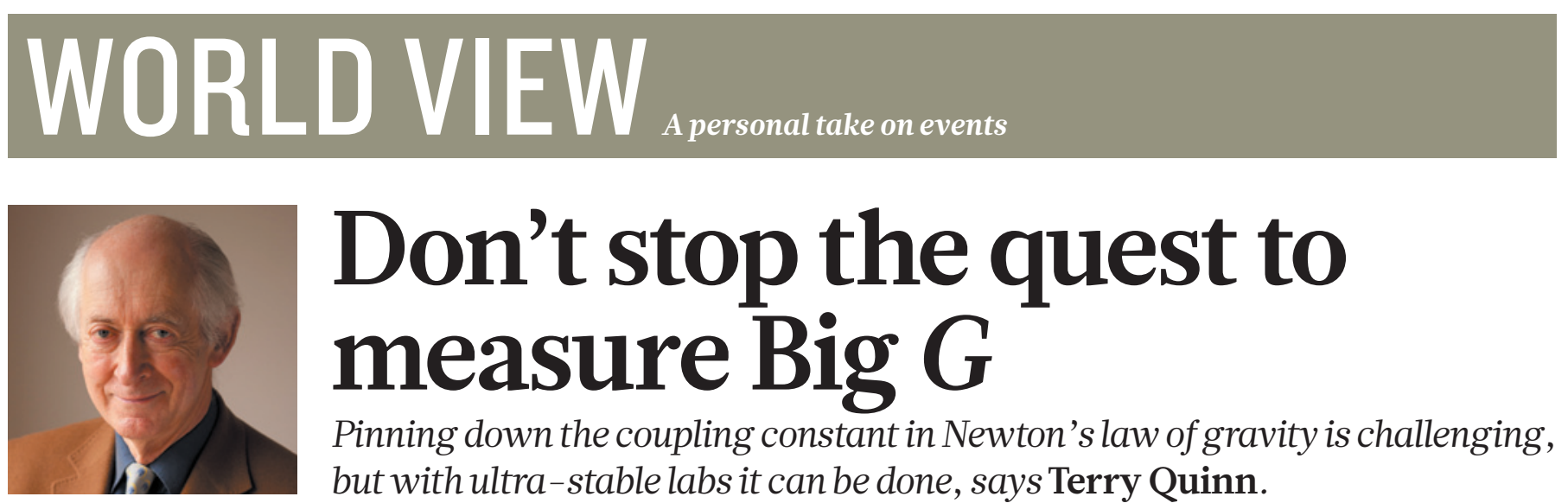

\title{
Don't stop the quest to measure Big $G$
}

Pinning down the coupling constant in Newton's law of gravity is challenging, but with ultra-stable labs it can be done, says Terry Quinn.

$\mathrm{P}$ lans are well under way to redefine the International System of Units (SI) by basing it on seven physical constants - the kilogram, for instance, is to be linked to the numerical value of Planck's constant, rather than the current definition of the mass of a platinum-iridium cylinder.

The seven constants help to explain and predict the motion and actions of the Universe. But one that is not included is what we in the business call Big G: the coupling constant in Newton's law of gravity.

There is a problem with $\operatorname{Big} G$ (so called to distinguish it from little $g$, the acceleration due to gravity at Earth's surface). Current measurements of it are, frankly, all over the place. Seven separate experiments in the past decade or so have given results that have a spread of about $0.05 \%$. For a fundamental constant of physics, that is extraordinarily imprecise.

This uncertainty has little impact on day-today life, and Einstein's general theory of relativity has long replaced Newton's law of gravity as the way for scientists to view physics at the largest scales. Yet Newton's law still predicts with adequate precision the movements of the planets and their moons, artificial satellites and space probes. (We don't need to isolate the value of $G$ to calculate these movements, because the equations depend on a combination of their masses and $G$.)

For a scientist - and a former director of the International Bureau of Weights and Measures (BIPM) in Paris such as myself - the imprecision in $G$ is irritating. Moreover, there is a solid scientific case for sorting it out. The search for a theory of quantum gravity that is consistent with quantum electrodynamics is perhaps the most active field of theoretical physics. One day, we may have to test such theories by comparing the values of $G$ that they predict with the real thing - so we need an accurate experimental value.

The problem for the physicist on Earth who tries to measure $G$ is that, although the strength of gravity is huge on an astronomical scale, it is extremely small in a laboratory. The force of gravity holds the planets in their orbits around the Sun and the billions of stars in the arms of the galaxies, yet this is the same gravitational force that, between a pair of 1-kilogram copper balls that are just touching, is about $10^{-8}$ times the weight of each.

To pick up this tiny signal, the laboratory itself has to be mechanically stable, with a low level of ground vibration and tilt, and with the temperature of its apparatus stabilized to a few thousandths of a degree Celsius.

Assuming that there is no hidden physics that can explain why the value of $G$ measured in different places would be different (unlikely), why

\section{DNATURE.COM}

Discuss this article online at:

go.nature.com/6wheyh is there such a spread of results? The problem lies in systematic error - the spectre that haunts every absolute determination of a fundamental constant. No matter how much one tries to take into account every possibility for error in a measurement, it is in principle impossible to demonstrate its absence. The only way to give confidence is to measure the same constant using a number of different methods. This is true in the measurement not only of a fundamental constant of nature, but of anything else.

At the BIPM, we devised an experiment to measure $G$ with two almost-independent methods in the same apparatus. Our results were at the high end of the range. They did not have the smallest level of uncertainty of any $G$ experiments, but they are the only ones that have been repeated and it is the only $G$ experiment in which more than one method has been used (we published the original in 2001 and then the follow-up last year).

There is another, more subtle, problem, which is related to the experimenter's behaviour. In measuring $G$, or any other constant, one starts out with a pretty good idea of its value. As the data come in, it starts to become clear roughly where the final result will lie. More data refine the value and correct both large and small errors. At what point does the experimenter stop searching for errors? There is an almost irresistible pressure to stop when the result is about what one expects it to be. Concealing the results from the experimenter solves this problem but sets up another crude errors are missed and thus waste valuable time and effort.

So it is difficult to find the true value of $G$, but I believe that it can be done. New efforts and new measurements are needed. Both the US National Institute of Standards and Technology in Gaithersburg, Maryland, and the UK National Physical Laboratory in Teddington have or are building ultra-stable metrology laboratories that offer the best chance yet to pin down Big G. I have already suggested to them that they carry out $G$ experiments - and, of course, I would like those to be improved versions of the BIPM experiment.

Is it worth it? The answer must be yes. I will make the case again at a meeting of physicists and metrology experts at the Royal Society in London next month, organized by me, Clive Speake of the University of Birmingham, UK, and Luo Jun of Huazhong University of Science \& Technology in Wuhan, China. The title of the meeting is 'The Newtonian constant of gravitation, a constant too difficult to measure?'. The answer to that is surely no.

Terry Quinn is emeritus director of the BIPM near Paris. e-mail: tjqfrs@gmail.com 